\title{
Lean mass, magnesium, faecal calprotectin and glucocorticoid exposure as risk factors for low bone mineral density in inflammatory bowel disease patients
}

Iulia Soare ${ }^{1}$, Anca Sirbu ${ }^{1,2}$, Mihai Mircea Diculescu ${ }^{1,3}$, Bogdan Radu Mateescu ${ }^{1,4}$, Cristian Tieranu ${ }^{1,5}$, Sorina Martin ${ }^{1,2}$, Carmen Gabriela Barbu ${ }^{1,2}$, Mirela Ionescu ${ }^{1,5}$ and Simona Fica ${ }^{1,2}$

'University of Medicine and Pharmacy 'Carol Davila' Bucharest, Bucharest, Romania

${ }^{2}$ Department of Endocrinology, Diabetes and Metabolic diseases, Elias Hospital, Bucharest, Romania

${ }^{3}$ Department of Gastroenterology, Fundeni Clinical Institute, Bucharest, Romania

${ }^{4}$ Department of Gastroenterology, Colentina Hospital, Bucharest, Romania

${ }^{5}$ Department of Gastroenterology, Elias Hospital, Bucharest, Romania

Correspondence should be addressed to A Sirbu: aesirbu12@gmail.com

\begin{abstract}
Background and aim: Low bone mineral density (BMD) is a common complication in patients with inflammatory bowel disease (IBD). However, debates are ongoing with regard to the other involved factors, especially in younger patients. This study aimed to evaluate the parameters that contribute to decreased BMD, focusing on premenopausal women and men aged $<50$ years.

Methods: This study included 81 patients with IBD and 81 age-, sex- and BMI-matched controls. Blood tests were conducted on IBD patients, and a dual-energy X-ray absorptiometry (DXA) scan was performed on both groups.

Results: Low BMD and fragility fracture were found to be more prevalent in IBD patients than in healthy subjects $(49.3 \%$ vs $23.4 \%, P=0.001$ and $9.8 \%$ vs $1.2 \%, P=0.01$, respectively). Patients with low BMD were older, with a longer disease duration, higher faecal calprotectin (FC) levels and lower magnesium and lean mass (appreciated as appendicular skeletal muscle index (ASMI)). Multiple regression analysis revealed that ASMI, age and use of glucocorticoids were the independent parameters for decreased BMD. Although $91.3 \%$ of the patients had a 25 -hydroxy vitamin $D$ level of $<30 \mathrm{ng} / \mathrm{mL}$, it was not a statistically significant factor for decreased BMD.

Conclusion: In our study, the levels of vitamin D did not seem to have an important impact on BMD. Conversely, FC, magnesium and lean mass are important factors, suggesting that good control of disease, adequate magnesium intake and increased lean mass can have a good impact on bone metabolism in patients with IBD.
\end{abstract}

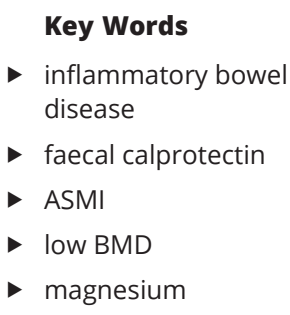

Endocrine Connections (2021) 10, 918-925

\section{Introduction}

Inflammatory bowel diseases (IBD), including Crohn's disease (CD) and ulcerative colitis (UC), have an unknown aetiology. Multiple factors, such as genetic predisposition, microbiota changes, environmental factors and autoimmune involvement, have been proposed. IBD is associated with decreased bone mineral density (BMD), with the prevalence of osteoporosis and osteopenia reported to be between 22 and $77 \%$, depending on https://ec.bioscientifica.com

https://doi.org/10.1530/EC-21-0138 (c) 2021 The authors Published by Bioscientifica Ltd

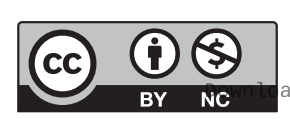

This work is licensed under a Creative Commons Attribution-NonCommercial 4.0 International License. ded from Bioscientifica.com at 04/26/2023 01:02:11PM 
the study (1). Sheth et al. found that the prevalence of osteopenia ranges from 32 to $36 \%$ and osteoporosis from 7 to $15 \%$ (2). Fracture risk is higher in these patients than in healthy subjects, according to a recent meta-analysis showing a $32 \%$ increased risk, which is consistent with different cohort studies previously conducted (3). Many factors are associated with decreased $\mathrm{BMD}$, one of which is the use of high-dose glucocorticoids. A Swiss IBD cohort study has reported an increased percentage of steroid use in IBD patients with osteoporosis (79.2\%) and osteopenia $(62.6 \%)$ and decreased percentage in patients with normal BMD (42.2\%) (4).

Although new biologic therapies have been developed and the use of high-dose glucocorticoids has declined, low BMD is still present in these patients, suggesting that other parameters may cause bone impairment. If vitamin D and calcium are traditional factors associated with decreased $\mathrm{BMD}$, low magnesium is a very common situation in IBD patients, especially in CD patients, with an intake of $60-63 \%$ of the daily requirement (5).

According to the ECCO guidelines, screening for low BMD is recommended for IBD patients similar to the general population, with a special focus on the patients' history of long-term corticosteroid treatment (>3 months) and fragility fracture history as well as their age (6). The British Society of Gastroenterology suggests screening according to the FRAX score in patients aged $>40$ years and based on multiple exposures to corticosteroid treatment in others (8).

This study aimed to evaluate the prevalence and the factors linked to low BMD in patients with IBD, with a special focus on premenopausal women and men aged $<50$ years. Demographic, clinical and paraclinical parameters have been studied along with dual-energy $\mathrm{X}$-ray absorptiometry (DXA) to identify patients at a high risk of having low BMD.

\section{Methods}

During a research project, patients from three IBD tertiary centres, namely, Fundeni, Elias and Colentina, in Bucharest were referred to our clinic for endocrinological evaluation. The cross-sectional study included 81 adult ( $>18$ years old) patients suffering from IBD and 81 BMI-, gender- and agematched healthy subjects.

A signed informed consent was obtained from the patients. The study adhered to the ethical principles of the Declaration of Helsinki and was approved by the Ethics Committee of Elias Hospital and University of Medicine and Pharmacy 'Carol Davila' Bucharest. The exclusion criteria included other secondary causes of low BMD (hyperparathyroidism, hyperthyroidism, renal disease or liver disease) and pregnancy. None of the patients or the subjects received calcium or vitamin D supplements or anti-osteoporotic treatment.

General information such as age, age of diagnosis, disease duration, intestinal resection/surgery history, current medications and glucocorticoid exposure were obtained from the patients' records.

Patient workup included anthropometric measurements (height and weight), calculation of the BMI, fasting blood tests (calcium, albumin and magnesium levels, ESR) and hormonal profile (parathyroid hormone, 25-hydroxy vitamin D). They were assayed at a single laboratory using enzyme-labelled chemiluminescent immunometric assay (Immulite 2000, Siemens Healthcare Diagnostics Products Ltd.). FC level was also documented via immunochromatography.

The albumin-adjusted calcium level was calculated using the following formula: corrected calcium $(\mathrm{mg} / \mathrm{dL})=$ measured total $\mathrm{Ca}(\mathrm{mg} / \mathrm{dL})+0.8$ (4.0-serum albumin $(\mathrm{g} / \mathrm{dL})$ ), where 4.0 is the average albumin level.

Vitamin D deficiency and insufficiency were defined as 25 -hydroxy vitamin D level of $<20 \mathrm{ng} / \mathrm{mL}$ and between 20 and $30 \mathrm{ng} / \mathrm{mL}$, respectively.

DXA using Lunar iDXA (ME+212018) was performed on all the participants in this study, with total hip, femoral neck and lumbar spine BMD (grams/ $\left.\mathrm{cm}^{2}\right)$, whole-body scan and vertebral fracture assessment (VFA). Trabecular bone score (TBS) was calculated for the same region as that in the LS BMD assessment using TBS version 3.0.2.0. All participants underwent VFA, and in those suspected to have lumbar spine fracture, X-ray or MRI confirmed the diagnosis.

According to the International Society of Clinical Densitometry official position (8), in premenopausal women and men aged $<50$ years, a Z score of $<-2$ s.D. at the hip or spine is defined as BMD below the expected range for age. In the other patients, the T-score was used for diagnosis, with a T-score below -2.5 S.D. and T-score $<=-2.5$ and $<-1$ S.D. indicating osteoporosis and osteopenia, respectively. Based on these criteria, the patients were divided into two groups: one group with normal BMD and the other with low BMD (Z score $<-2$ s.D. in premenopausal women and men $<50$ years and $T$ score $<-1$ s.D. in the others).

Glucocorticoid exposure was quantified as no exposure (less than 3 months) or positive exposure (more than 3 months, more than $7.5 \mathrm{mg} /$ day of prednisone). 
To evaluate lean mass, according to the European Working Group on Sarcopenia in Older People and the International Working Group on Sarcopenia, the appendicular skeletal muscle index (ASMI) was used and calculated as the sum of muscle mass on the legs and arms divided by square height (ASM/height $\left.{ }^{2}\right)(9,10)$. Myopenia was defined as Z score <-1 DS (9) with population-based and age- and gender-matched data values from the National Health and Nutrition Examination Survey (NHANES) (11).

\section{Statistical analysis}

Statistical analysis was conducted using SPSS version 21, and continuous variables are expressed as mean \pm S.D. and median and interquartile range (IQR) for normal distribution and nonparametric distribution, respectively. Differences in biochemical and DXA parameters were calculated using a nonparametric test: Mann-Whitney $U$-test or independent samples $t$-test. The associations between pairs of variables were calculated using the chi-squared test $\left(\chi^{2}\right)$. The validity of the parameters was measured using the area under the receiver operating characteristic (ROC) curve with 95\% CI. A binary logistic regression model was used to assess the influence of different parameters on the presence of low BMD. Two-sided $P$-value of $<0.05$ was considered statistically significant.

\section{Results}

In this study, 81 patients (48 and 33 patients diagnosed with CD and UC, respectively) were evaluated. Of them, 38 (46.9\%) were men, and 43 (53.1\%) were women. Their median age was 43 (IQR 24) years; mean age at diagnosis, $32( \pm 14.5)$ years and median duration of the disease, 7 (IQR 10) years. A total of 81 healthy subjects were included and matched for BMI, gender and age.
Low BMD was observed in 40 patients (49.3\%) and was more prevalent in CD patients than in UC patients $(56.2 \%$ vs $39.3 \%, \chi^{2}=2.2, P=0.1$ ) as well as in female than in male patients ( $55.8 \%$ vs $\left.42.1 \%, \chi^{2}=1.5, P=0.2\right)$. Moreover, when CD patients were compared with UC patients, no statistical differences were observed in terms of demographic data, DXA parameters or prevalence of low BMD and myopenia. The data are presented in Table 1.

In the group of healthy subjects, low BMD was observed in 19 patients, with a decreased prevalence compared with IBD patients $(23.4 \%$ vs $49.3 \%, P=0.001)$. BMD below the range for age and gender was found in 17 IBD patients and 6 controls $(32.69 \%$ vs $11.3 \%, P=0.01)$. In postmenopausal women and men aged $>50$ years, osteopenia was observed in 12 IBD patients (out of 29) vs 11 healthy subjects (out of 23) (41.3\% vs 47.8\%), whereas osteoporosis was observed in 11 vs 2 (37.9\% vs $8.6 \%$, $P=0.009)$. Of the IBD patients, $8(9.87 \%)$ already had fragility fracture ( 5 of them were diagnosed with vertebral fractures during the study period) vs the group of controls, where one of them was diagnosed with vertebral fracture (9.8\% vs $1.2 \%, P=0.01$ ).

\section{Factors associated with low BMD in patients with IBD}

The patients with low BMD were older (median age: 51 (25) vs 34 (19) years, $P<0.005)$, with a later onset of the disease ( $37 \pm 14$ vs $30 \pm 13.4$ years, $P=0.03)$, longer duration of the disease $(8.5$ (12) vs 5 (7) years, $P=0.03)$ and an increased level of FC (100 (270) vs $31(120) \mu \mathrm{g} / \mathrm{g}, P<0.05)$. Magnesium was statistically lower in the low BMD group than in the normal BMD group $(1.94 \pm 0.1$ vs $2.02 \pm 0.12$ $\mathrm{mg} / \mathrm{dL}, P=0.03)$. Although vitamin $\mathrm{D}$ deficiency and insufficiency were observed in 38 and 36 patients $(91.3 \%$ of all patients), respectively, the levels of 25-hydroxy

Table 1 Demographic, laboratory and DXA characteristics of the patients with IBD, according to the type of disease.

\begin{tabular}{lcc}
\hline Parameter & All patients (81) \\
\hline Age, years & $43(24)$ \\
Age at diagnosis, years & $32 \pm 14.5$ \\
Disease duration, years & $7(10)$ \\
25-hydroxy vitamin D, ng/mL & $20.7 \pm 7.6$ \\
Lumbar BMD, g/cm² & $1.06 \pm 0.18$ \\
Hip BMD, g/cm² & $0.88 \pm 0.13$ \\
TBS & $1.38 \pm 0.1$ \\
Low BMD, \% & $40(49.3)$ \\
Myopenia, \% & $33(40.7)$ \\
\hline
\end{tabular}

\begin{tabular}{c}
\hline CD patients $(48)$ \\
\hline $42(23)$ \\
$34.1 \pm 14.9$ \\
$8(6)$ \\
$19.9 \pm 7.6$ \\
$1.04 \pm 0.18$ \\
$0.87 \pm 0.14$ \\
$1.38 \pm 0.1$ \\
$27(56.2)$ \\
$22(45)$ \\
\hline
\end{tabular}

\begin{tabular}{ccc}
\hline UC patients $(33)$ & & P-value \\
\cline { 1 - 1 } $34(27)$ & & NS \\
$33.8 \pm 14$ & & NS \\
$10.3(13)$ & & NS \\
$21.4 \pm 7.04$ & NS \\
$1.09 \pm 0.17$ & NS \\
$0.91 \pm 0.1$ & NS \\
$1.38 \pm 0.1$ & NS \\
$13(39.3)$ & NS \\
$11(33.3)$ & & \\
\hline
\end{tabular}

Values are presented as mean \pm S.D. or median (IQR), percentage, according to the type of variable and the normality of distribution. NS, not significant, $P$ value $>0.05$

ASMI, appendicular skeletal muscular index; BMD, bone mineral density; ESR, erythrocyte sedimentation rate; TBS trabecular bone score.

https://ec.bioscientifica.com

https://doi.org/10.1530/EC-21-0138 (c) 2021 The authors Published by Bioscientifica Ltd

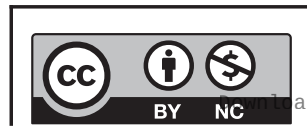

This work is licensed under a Creative Commons Attribution-NonCommercial 4.0 International License. ded from Bioscientifica.com at 04/26/2023 01:02:11PM via free access 
vitamin $\mathrm{D}(19.9 \pm 9.1$ vs $21.1 \pm 7.67, P=0.4)$ were not significantly different in the low BMD group and normal BMD group. Moreover, no significant difference was observed in the albumin-adjusted level of calcium $(8.9(0.4)$ vs $9.03(0.3) \mathrm{mg} / \mathrm{dL}, P=0.75)$ between the two groups.

ASMI was lower in patients with decreased BMD than in those with normal BMD $\left(6.58 \pm 1.6\right.$ vs $7.19 \pm 1.15 \mathrm{~kg} / \mathrm{m}^{2}$, $P=0.03)$. Myopenia was observed in 33 patients with IBD, of whom 21 and 12 were diagnosed with low BMD and normal BMD, respectively $\left(52.5 \%\right.$ vs $29.2 \%, \chi^{2}=4.17$, $P=0.03)$. In the group having patients with decreased BMD, a statistically significant difference was observed among those with glucocorticoid exposure $(87.5 \%$ vs $65.4 \%, \chi 2=6.3, P=0.01)$.

The data are presented in Table 2 .

The ability of each parameter to differentiate normal BMD from low BMD was medium, with the best area under the ROC curve for the level of ASMI and magnesium $(0.654, P=0.01$, and $0.645, P=0.02$, respectively) (Fig. 1).

In multiple logistic regression analysis, age $(\beta=0.51$, $P=0.02)$, glucocorticoid exposure $(\beta=-1.45, P=0.02)$ and ASMI $(\beta=-0.939, P=0.03)$ were the only independent factors of low BMD in a model that included BMI and gender.

\section{Low BMD distribution in men and women}

The differences between women and men in the presence of decreased BMD are presented in Table 3. For females, factors such as age and magnesium level were significantly different between the two groups. In males, the only parameter that seemed to influence the presence of low BMD was ASMI.

When analysed in subgroups, ASMI was statistically associated with low BMD (AUROC $=0.717, P=0.025)$, in men, but not in women (Fig. 2).

\section{Determinants of low BMD in premenopausal women and men aged $<50$ years}

When the group of premenopausal women and men aged $<50$ years was investigated, 17 out of 52 patients (32.69\%) had low BMD, and 2 of them, 23 and 33 years old, had a vertebral fragility fracture. When the two groups were compared, FC and ASMI were statistically different (150 (402) vs $30(105) \mu \mathrm{g} / \mathrm{g}, P<0.005$, and $6.27 \pm 1.11 \mathrm{vs}$ $6.71 \pm 1.3 \mathrm{~kg} / \mathrm{m}^{2}, P=0.045$, respectively); a higher index of intestinal inflammation and low mean mass were the most important parameters associated with low BMD, as presented in Table 4.

When applied in premenopausal women and men aged $<50$ years, the level of FC seemed to be associated with low BMD (AUROC $=0.735, P=0.006)$ (Fig. 3).

\section{Discussion}

Our study addressed the prevalence and factors associated with low BMD, confirming the low level of awareness of the decreased BMD in premenopausal women and men aged $<50$ years.

Table 2 Demographic, laboratory and DXA characteristics of the patients with IBD, according to the presence of low BMD.

\begin{tabular}{lcc}
\hline Parameter & All patients $(81)$ \\
\hline Age, years & $43(24)$ \\
Age at diagnosis, years & $32 \pm 14.5$ \\
Disease duration, years & $7(10)$ \\
ESR, mm/h & $13(11)$ \\
FC, $\mathrm{gg} / \mathrm{g}$ & $55(220)$ \\
25-hydroxy vitamin D, ng/mL & $20.7 \pm 7.6$ \\
Albumin-adjusted level of calcium, mg/dL & $9.02(0.6)$ \\
Magnesium, mg/dL & $1.99 \pm 0.1$ \\
ASMI, kg/m² & $6.89 \pm 1.22$ \\
Myopenia, \% & $33(40.7)$ \\
Lumbar BMD, g/cm² & $1.06 \pm 0.18$ \\
Hip BMD, g/cm² & $0.88 \pm 0.13$ \\
TBS & $1.38 \pm 0.1$ \\
Glucocorticoid exposure, \% & $61(75.3)$ \\
\hline
\end{tabular}

\begin{tabular}{c}
\hline Low BMD $(40)$ \\
\hline $51(25)$ \\
$37 \pm 14$ \\
$8.5(12)$ \\
$14(13)$ \\
$100(270)$ \\
$19.9 \pm 9.1$ \\
$8.9(0.4)$ \\
$1.94 \pm 0.1$ \\
$6.58 \pm 1.6$ \\
$21(52.5)$ \\
$0.94 \pm 0.1$ \\
$0.81 \pm 0.1$ \\
$1.32 \pm 0.1$ \\
$35(87.5)$ \\
\hline
\end{tabular}

\begin{tabular}{cccc}
\hline Normal BMD $(41)$ & & P-value \\
\cline { 1 - 1 } $34(19)$ & & $<0.005$ \\
$30 \pm 13.4$ & & 0.03 \\
$5(7)$ & & 0.03 \\
$10(8)$ & & $N S$ \\
$31(120)$ & & $<0.05$ \\
$21.1 \pm 7.67$ & & $N S$ \\
$9.03(0.3)$ & & $N S$ \\
$2.02 \pm 0.12$ & & 0.03 \\
$7.19 \pm 1.15$ & & 0.02 \\
$12(29.2)$ & & 0.03 \\
$1.17 \pm 0.1$ & & $<0.001$ \\
$0.95 \pm 0.1$ & & $<0.001$ \\
$1.43 \pm 0.08$ & & $<0.001$ \\
$26(65.4)$ & & 0.01
\end{tabular}

Values are presented as mean \pm S.D. or median (IQR), percentage, according to the type of variable and the normality of distribution. $P$-value statistically significant differences $P<0.05$.

ASMI, appendicular skeletal muscular index; BMD, bone mineral density; ESR, erythrocyte sedimentation rate; FC, faecal calprotectin; TBS trabecular bone score.

https://ec.bioscientifica.com

https://doi.org/10.1530/EC-21-0138 (c) 2021 The authors Published by Bioscientifica Ltd

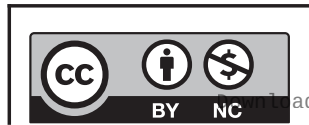

This work is licensed under a Creative Commons Attribution-NonCommercial 4.0 International License. ded from Bioscientifica.com at 04/26/2023 01:02:11PM 


\section{ROC Curve}

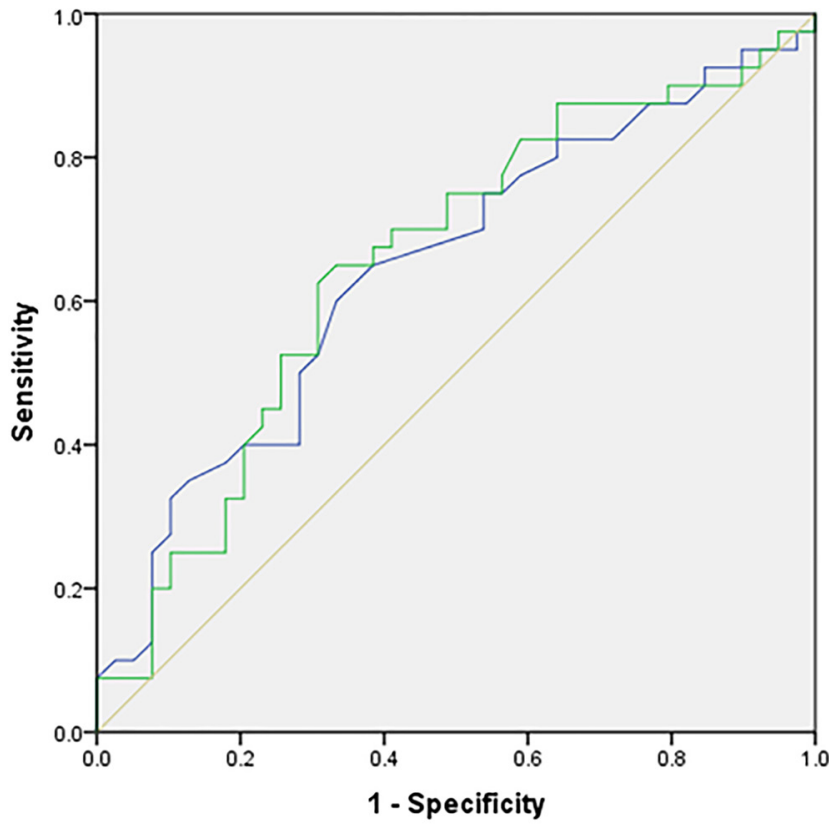

ROC Curve (Area)

Magnesium (0.645)

ASMI (0.654)

Figure 1

Predictive values of magnesium, ASMI for diminishing between low and normal BMD in IBD patients.

Although the prevalence was high, only seven of the patients were previously screened, and five of them who were diagnosed with osteoporosis received specific treatment (oral bisphosphonates, but gastrointestinal intolerance was reported, and the treatment was stopped). Moreover, eight of the patients had a fragility fracture. The prevalence of low BMD and fragility fracture was found to be higher than in healthy subjects. This emphasises the fact that decreased BMD and fragility fractures are a real problem in IBD patients, even in younger adults, sometimes neglected. For the Romanian population, a previous study, which included 47 patients with IBD from the northern part of Romania, demonstrated a prevalence of $48.07 \%$ and $56.41 \%$ in patients with UC and with CD (12). In another study conducted in Romania, which included 188 IBD patients (74.4\% with UC and $25.55 \%$ with $\mathrm{CD}$ ), osteopenia and osteoporosis were observed in $48.5 \%$ and $15.2 \%$, respectively, in CD and $56.5 \%$ and $17.19 \%$, respectively, in CU (13). A study (14) conducted in Poland demonstrated a high prevalence of osteopenia and osteoporosis in women $(43.4,45.3 \%)$ and men $(38.2$, $24.5 \%$ ), more common in CD than in UC patients. A recent meta-analysis of 16 studies revealed an increased risk for fragility fracture, especially in the spine, emphasising the need to identify patients at risk (15).

One of the most important determinants of decreased BMD in IBD patients is exposure to a high dose of glucocorticoid, which agrees with the findings of this study. Several studies pointed out their role on bone impairment, with a recent study (16) demonstrating that the use of glucocorticoids increased the risk for osteoporosis (LR=5.1, $P=0.028)$.

Moreover, our study revealed that longer disease duration has a negative impact on the bone, as demonstrated in previous studies (14), possibly due to ageing and increased exposure to glucocorticoids.

Evaluation of vitamin D is important in low BMD, due to its important role in bone mineralization. However, the association between 25-hydroxy vitamin D and BMD is on debate $(17,18)$.

Table 3 Clinical, laboratory and DXA characteristics of the patients with IBD, according to their gender.

\begin{tabular}{|c|c|c|}
\hline Gender & Parameter & All (38) \\
\hline \multirow[t]{7}{*}{ Male (38) } & ASMI, kg/m² & $7.52 \pm 1.26$ \\
\hline & Age, years & $35.5(20)$ \\
\hline & Magnesium, mg/dL & $2.01 \pm 0.1$ \\
\hline & $\mathrm{BMI}, \mathrm{kg} / \mathrm{m}^{2}$ & $23.4(5.5)$ \\
\hline & 25-hydroxy vitamin $\mathrm{D}, \mathrm{ng} / \mathrm{dL}$ & $20.4 \pm 7.4$ \\
\hline & $\mathrm{FC}, \mu \mathrm{g} / \mathrm{g}$ & $100(243)$ \\
\hline & Parameter & All (43) \\
\hline \multirow[t]{5}{*}{ Female (43) } & ASMI, kg/m² & $6.34 \pm 0.9$ \\
\hline & Age, years & $47(25)$ \\
\hline & Magnesium, mg/dL & $1.98 \pm 0.1$ \\
\hline & 25-hydroxy vitamin $\mathrm{D}, \mathrm{ng} / \mathrm{dL}$ & $20.9 \pm 7.9$ \\
\hline & $\mathrm{FC}, \mu \mathrm{g} / \mathrm{g}$ & $30(155)$ \\
\hline
\end{tabular}

\begin{tabular}{c}
\hline Low BMD (16) \\
\hline $6.95 \pm 1.54$ \\
$44(26)$ \\
$1.98 \pm 0.1$ \\
$22.6(4.26)$ \\
$20 \pm 0.6$ \\
$175(263)$ \\
\hline Low BMD $(24)$ \\
\hline $6.34 \pm 0.9$ \\
$54(30)$ \\
$1.94 \pm 0.1$ \\
$19.3 \pm 8.6$ \\
$41(277)$ \\
\hline
\end{tabular}

\begin{tabular}{|c|c|}
\hline Normal BMD (22) & P-value \\
\hline $7.96 \pm 0.8$ & 0.01 \\
\hline $32(17)$ & NS \\
\hline $2.02 \pm 0.1$ & NS \\
\hline $23.5(7.2)$ & NS \\
\hline $20 \pm 0.7$ & NS \\
\hline $62(138)$ & NS \\
\hline Normal BMD (19) & $P$-value \\
\hline $6.34 \pm 0.87$ & NS \\
\hline $35(19)$ & 0.001 \\
\hline $2.03 \pm 0.1$ & 0.02 \\
\hline $22.2 \pm 6.9$ & NS \\
\hline $15(105)$ & NS \\
\hline
\end{tabular}

Values are presented as mean \pm S.D. or median (IQR), according to the type of variable and the normality of distribution. $P$-value statistically significant differences $P<0.05$.

ASMI, appendicular skeletal muscular index; FC, faecal calprotectin. 
ROC Curve

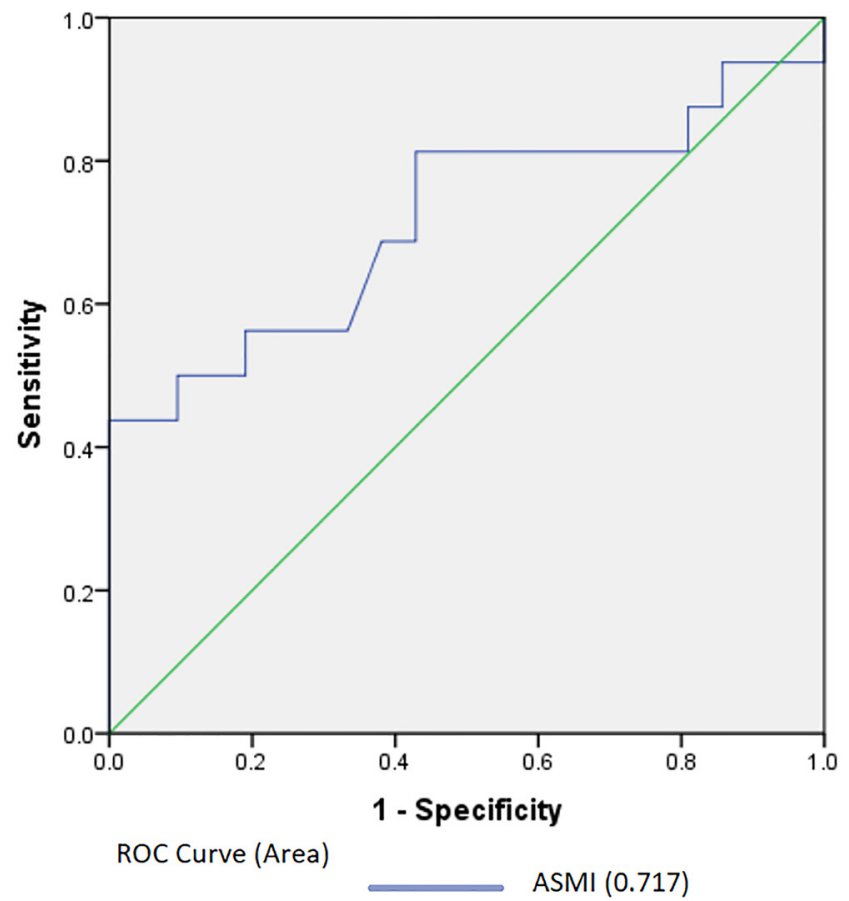

Figure 2

Predictive values of ASMI for diminishing between low and normal BMD in men with IBD.

Vitamin D level is a parameter that is low in IBD patients, due to multiple reasons, including malabsorption, malnutrition and low sunlight exposure. In our study, a low vitamin D level was observed in $91 \%$ of the patients, but it had no impact on BMD. Similar data were obtained from other studies, Pintilie's conducted on the Romanian population (10) and Even's (16), showing that 25-hydroxy vitamin D did not correlate with BMD in IBD patients However, mean vitamin D was borderline insufficiency and deficit, which could have influenced the results of BMD, due to osteomalacia.

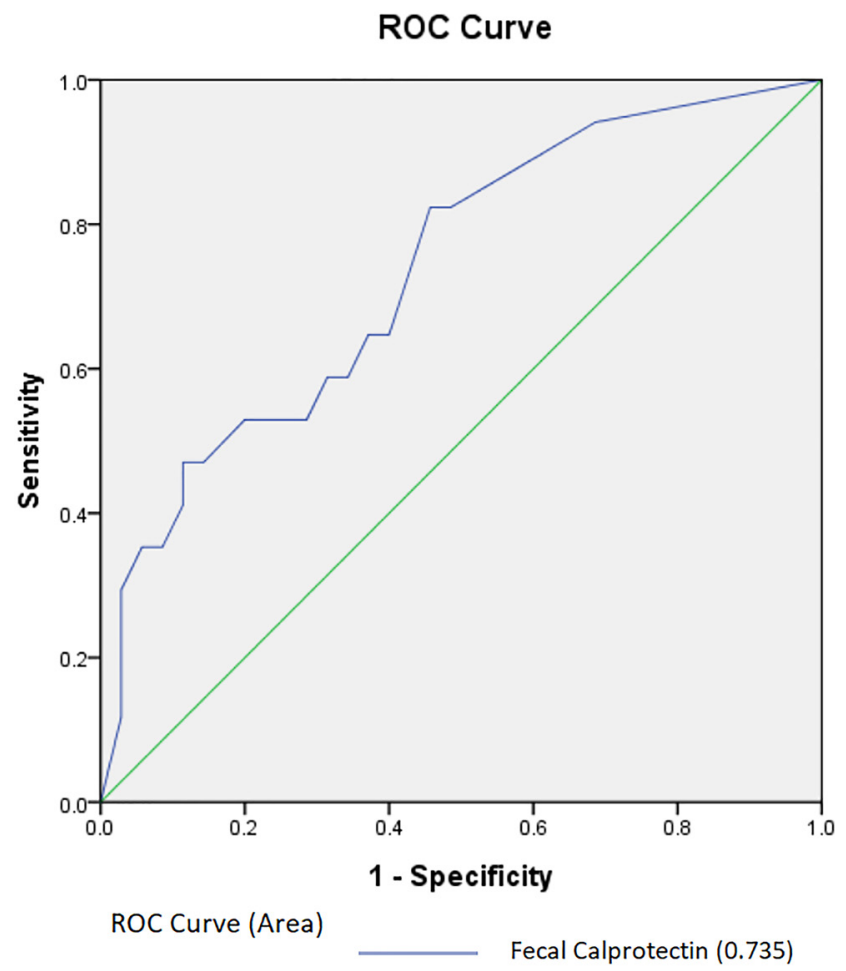

Figure 3

Predictive values of fecal calprotectin for diminishing between low and normal BMD in premenopausal women and men $<50$ years with IBD.

The important role of magnesium in bone formation is often neglected. Low level of magnesium directly inhibits osteoblast activity while increasing osteoclast activity (19). Moreover, studies have demonstrated the relationship between inflammation and magnesium, with the levels of TNFa, IL1a and IL6 being increased in patients with hypomagnesaemia (20). Our study demonstrated that low magnesium is a good predictor of low BMD. In terms of gender, magnesium seemed to have a significant impact on women; this may be because the women included in our study were older than the men and had a higher prevalence

Table 4 Demographic, clinical, laboratory and DXA parameters premenopausal women and men <50 years IBD patients.

\begin{tabular}{l} 
Parameter \\
\hline Age, years \\
Age at diagnosis, years \\
Disease duration, years \\
FC, $\mu$ g/g \\
25-hydroxy vitamin D, ng/mL \\
Albumin-adjusted calcium, mg/dL \\
ASMI, kg/m² \\
BMI, kg/m²
\end{tabular}

\begin{tabular}{c}
\hline All $(52)$ \\
\hline $32(16)$ \\
$26 \pm 10.1$ \\
$5(5)$ \\
$50(135)$ \\
$21.2 \pm 7.8$ \\
$8.96(0.56)$ \\
$6.8 \pm 1.3$ \\
$21.8(4.52)$ \\
\hline
\end{tabular}

\begin{tabular}{c}
\hline Low BMD (17) \\
\hline $31(20)$ \\
$26 \pm 11.7$ \\
$5(5)$ \\
$150(402)$ \\
$20.6 \pm 8.9$ \\
$8.92(0.6)$ \\
$6.27 \pm 1.11$ \\
$20.8(3.15)$
\end{tabular}

\begin{tabular}{c}
\hline Normal BMD $(35)$ \\
\hline $33(14)$ \\
$27.2 \pm 9$ \\
$5(8)$ \\
$30(105)$ \\
$21.6 \pm 6.9$ \\
$9.03(0.54)$ \\
$6.71 \pm 1.3$ \\
$22.2(5.89)$
\end{tabular}

\begin{tabular}{l}
\hline P-value \\
\hline NS \\
NS \\
NS \\
$<0.005$ \\
NS \\
NS \\
$<0.05$ \\
NS \\
\hline
\end{tabular}

Values are presented as mean \pm S.D. or median (IQR), according to the type of variable and the normality of distribution. $P$-value statistically significant differences $P<0.05$.

ASMI, appendicular skeletal muscular index; FC, faecal calprotectin.

https://ec.bioscientifica.com

https://doi.org/10.1530/EC-21-0138 (c) 2021 The authors Published by Bioscientifica Ltd

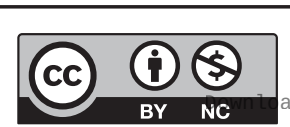

This work is licensed under a Creative Commons Attribution-NonCommercial 4.0 International License. ded from Bioscientifica.com at 04/26/2023 01:02:11PM 
of low BMD. A recent meta-analysis (21) also indicated an insignificant association between magnesium intake and BMD at the hip. To the best of our knowledge, there are no studies focusing on this association in IBD patients; further studies with a long-term follow-up should be conducted.

Although some studies demonstrated a possible association between low BMD and disease activity and severity, to the best of our knowledge, this is the first study to show the association between FC as a marker of intestinal inflammation and BMD, emphasising the already-known impact of chronic inflammation on the bone. FC is a useful marker of intestinal inflammation, and studies have demonstrated a strong correlation between them and endoscopic activity active disease in CD and UC $(22,23)$. A recent study by Lima et al. (24), which included 68 patients with UC and 60 with CD (aged 17-40 years), showed that disease activity was associated with osteopenia. Another study conducted by Cravo et al. (25) on 99 CD patients described a direct and significant association between disease activity and osteoporosis. Moreover, Abraham et al. (26) demonstrated that BMD is affected at diagnosis, suggesting that chronic inflammation could lead to decreased BMD.

One of the most important findings in our study was the relationship between lean mass and the risk for low BMD in IBD patients, which is independent of other parameters. Low BMI is a well-known factor for low BMD, as described in previous studies (27). Moreover, decreased lean mass, BMI and the presence of sarcopenia were identified as predictors of osteopenia and osteoporosis in IBD patients (28). Our study demonstrated that low ASMI, not BMI, could be a good predictor of low BMD, especially in younger patients. Moreover, myopenia is increased in IBD patients with decreased BMD. Considering the results in our study, a simultaneous whole-body scan when performing DXA could give us important information on muscle mass and help us identify the patients at risk of having low BMD, especially younger patients. Further studies should include the effect of regular physical activity on BMD in these patients.

The strength of this study is the availability of biochemical markers to assess both blood tests (ESR, calcium, albumin and 25-hydroxy vitamin D), intestinal inflammation (FC), DXA and whole-body scan in order to assess all the possible parameters that could affect BMD.

This study has several limitations, which include the relatively small number of patients, the heterogenic group of patients and the inclusion of young and older patients. Conversely, this could be one of the strengths of the study, as previous data were reported on paediatric or postmenopausal and older patients. To eliminate the confounding factors, the patients were divided into subgroups based on age or gender. In addition, as this is a cross-sectional study, we were not able to make the study at the time of diagnosis to see if there are precocious modifications in these patients. Another limitation of the study was that, due to the heterogeneous group and multiple therapies, the patients could not be divided according to their therapies. A prospective comparative study will help us characterise better the impact of the different therapies on BMD.

Our study confirmed that, although underdiagnosed in IBD patients, especially in younger patients, low BMD and fragility fracture are common, and screening should be performed to decrease the risk of complications. Wellknown factors, such as older age and glucocorticoid exposure, but also neglected factors, such as magnesium and lean mass, are important parameters in the differentiation between normal and decreased BMD. FC can be a good predictor of low BMD in premenopausal women and men aged $<50$ years. Multiple regression analysis revealed that ASMI, age and glucocorticoid exposure are independent determinants of low BMD in patients with IBD. Better control of the disease, increase in muscular mass and adequate magnesium intake could improve the BMD of these patients. To validate our results, a further prospective analysis should be conducted.

\section{Declaration of interest}

The authors declare that there is no conflict of interest that could be perceived as prejudicing the impartiality of the research reported.

\section{Funding}

This work did not receive any specific grant from any funding agency in the public, commercial or not-for-profit sector.

\section{Author contribution statement}

I S conceived the design development, performed the statistical analysis, interpreted the results, A S participate in the conception, analysis, interpretation of the data, M M D, B R M, C T, M I referred the patients, assisted in collecting the data and drafted the manuscript, S M, C G B developed the design, participate in data collection, S F supervised data collection and interpretation. All authors critically revised the manuscript, approved the final version and agree to be accountable for all the aspects of the work.

\section{Acknowledgement}

The authors thank all the patients who agreed to participate in this research study.

\section{References}

1 Ali T, Lam D, Bronze MS \& Humphrey MB. Osteoporosis in inflammatory bowel disease. American Journal of Medicine 2009122 599-604. (https://doi.org/10.1016/j.amjmed.2009.01.022) 
2 Sheth T, Pitchumoni CS \& Das KM. Musculoskeletal manifestations in inflammatory bowel disease: a revisit in search of immunopathophysiological mechanisms. Journal of Clinical Gastroenterology 201448 308-317. (https://doi.org/10.1097/ MCG.0000000000000067)

3 Hidalgo DF, Boonpheng B, Phemister J, Hidalgo J \& Young M. Inflammatory bowel disease and risk of osteoporotic fractures: a metaanalysis. Cureus 201911 e5810. (https://doi.org/10.7759/cureus.5810)

4 Schüle S, Rossel JB, Frey D, Biedermann L, Scharl M, Zeitz J, FreitasQueiroz N, Kuntzen T, Greuter T, Vavricka SR, et al. Widely differing screening and treatment practice for osteoporosis in patients with inflammatory bowel diseases in the Swiss IBD cohort study. Medicine 201796 e6788. (https://doi.org/10.1097/MD.0000000000006788)

5 Taylor L, Almutairdi A, Shommu N, Fedorak R, Ghosh S, Reimer RA, Panaccione R \& Raman M. Cross-sectional analysis of overall dietary intake and Mediterranean dietary pattern in patients with Crohn's disease. Nutrients 201810 1761. (https://doi.org/10.3390/nu10111761)

6 Harbord M, Annese V, Vavricka SR, Allez M, Barreiro-de Acosta M, Boberg KM, Burisch J, De Vos M, De Vries AM, Dick AD, et al. The first European evidence-based consensus on extra-intestinal manifestations in inflammatory bowel disease. Journal of Crohn's and Colitis 201610 239-254. (https://doi.org/10.1093/ecco-jcc/jjv213)

7 Lamb CA, Kennedy NA, Raine T, Hendy PA, Smith PJ, Limdi JK, Hayee B, Lomer MCE, Parkes GC \& Selinger C. British Society of Gastroenterology consensus guidelines on the management of inflammatory bowel disease in adults. Gut 201968 (Supplement 3) s1-s106. (https://doi.org/10.1136/gutjnl-2019-318484).

8 Shuhart CR, Yeap SS, Anderson PA, Jankowski LG, Lewiecki EM, Morse LR, Rosen HN, Weber DR, Zemel BS \& Shepherd JA. Executive Summary of the 2019 ISCD Position Development Conference on Monitoring Treatment, DXA Cross-calibration and Least Significant Change, Spinal Cord Injury, Peri-prosthetic and Orthopedic Bone Health, Transgender Medicine, and Pediatrics. Journal of Clinical Densitometry 201922 453-471. (https://doi.org/10.1016/j.jocd.2019.07.001)

9 Cruz-Jentoft AJ, Baeyens JP, Bauer JM, Boirie Y, Cederholm T, Landi F, Martin FC, Michel JP, Rolland Y, Schneider SM, et al. Sarcopenia: European consensus on definition and diagnosis: report of the European Working Group on Sarcopenia in older people. Age and Ageing 201039 412-423. (https://doi.org/10.1093/ageing/afq034)

10 Fielding RA, Vellas B, Evans WJ, Bhasin S, Morley JE, Newman AB, Abellan van Kan G, Andrieu S, Bauer J, Breuille D, et al. Sarcopenia: an undiagnosed condition in older adults. Current consensus definition: prevalence, etiology, and consequences. International working group on sarcopenia. Journal of the American Medical Directors Association 2011 12 249-256. (https://doi.org/10.1016/j.jamda.2011.01.003)

11 Kelly TL, Wilson KE \& Heymsfield SB. Dual energy X-ray absorptiometry body composition reference values from NHANES. PLOS ONE 20094 e7038. (https://doi.org/10.1371/journal.pone.0007038)

12 Dumitrescu G, Mihai C, Dranga M \& Prelipcean CC. Serum 25-hydroxyvitamin D concentration and inflammatory bowel disease characteristics in Romania. World Journal of Gastroenterology 201420 2392-2396. (https://doi.org/10.3748/wjg.v20.i9.2392)

13 Pintilie IA, Nedelciuc O, Blaj AM, Mihai C \& Cijevschi Prelipcean C. Bone density and bone metabolism in patients with inflammatory bowel disease. Journal of Crohn's and Colitis 20137 (Supplement_1) S126-S127. (https://doi.org/10.1016/S1873-9946(13)60314-1)

14 Krela-Kaźmierczak I, Michalak M, Szymczak-Tomczak A, ŁykowskaSzuber L, Stawczyk-Eder K, Waszak K, Kucharski MA, Dobrowolska A \& Eder P. Prevalence of osteoporosis and osteopenia in a population Region. Polish Archives of Internal Medicine 2018128 447-454. (https:// doi.org/10.20452/pamw.4292) of patients with inflammatory bowel diseases from the Wielkopolska

15 Szafors P, Che H, Barnetche T, Morel J, Gaujoux-Viala C, Combe B \& Lukas C. Risk of fracture and low bone mineral density in adults with inflammatory bowel diseases. A systematic literature review with meta-analysis. Osteoporosis International 201829 2389-2397. (https:// doi.org/10.1007/s00198-018-4586-6)

16 Even Dar R, Mazor Y, Karban A, Ish-Shalom S \& Segal E. Risk factors for low bone density in inflammatory bowel disease: use of glucocorticoids, low body mass index, and smoking. Digestive Diseases 201937 284-290. (https://doi.org/10.1159/000496935)

17 Bischoff-Ferrari HA, Dietrich T, Orav EJ \& Dawson-Hughes B. Positive association between 25-hydroxy vitamin D levels and bone mineral density: a population-based study on younger and older adults. American Journal of Medicine 2004116 634-639. (https://doi. org/10.1016/j.amjmed.2003.12.029)

18 Hannan MT, Litman HJ, Araujo AB, McLennan CE, McLean RR, McKinlay JB, Chen TC \& Holick MF. Serum 25-hydroxyvitamin D and bone mineral density in a racially and ethnically diverse group of men. Journal of Clinical Endocrinology and Metabolism 200893 40-46. (https://doi.org/10.1210/jc.2007-1217)

19 Orchard TS, Larson JC, Alghothani N, Bout-Tabaku S, Cauley JA, Chen Z, LaCroix AZ, Wactawski-Wende J \& Jackson RD. Magnesium intake, bone mineral density, and fractures: results from the Women's Health Initiative Observational Study. American Journal of Clinical Nutrition 201499 926-933. (https://doi.org/10.3945/ajcn.113.067488)

20 Mazur A, Maier JA, Rock E, Gueux E, Nowacki W \& Rayssiguier Y. Magnesium and the inflammatory response: potential physiopathological implications. Archives of Biochemistry and Biophysics 2007458 48-56. (https://doi.org/10.1016/j.abb.2006.03.031)

21 Farsinejad-Marj M, Saneei P \& Esmaillzadeh A. Dietary magnesium intake, bone mineral density and risk of fracture: a systematic review and meta-analysis. Osteoporosis International 201627 1389-1399. (https://doi.org/10.1007/s00198-015-3400-y)

22 Schoepfer AM, Beglinger C, Straumann A, Trummler M, Renzulli P \& Seibold F. Ulcerative colitis: correlation of the Rachmilewitz endoscopic activity index with fecal calprotectin, clinical activity, C-reactive protein, and blood leukocytes. Inflammatory Bowel Diseases 200915 1851-1858. (https://doi.org/10.1002/ibd.20986)

23 Sipponen T, Kärkkäinen P, Savilahti E, Kolho KL, Nuutinen H, Turunen U \& Färkkilä M. Correlation of faecal calprotectin and lactoferrin with an endoscopic score for Crohn's disease and histological findings. Alimentary Pharmacology and Therapeutics 2008 28 1221-1229. (https://doi.org/10.1111/j.1365-2036.2008.03835.x)

24 Lima CA, Lyra AC, Mendes CMC, Lopes MB, Coqueiro FG, Rocha R \& Santana GO. Bone mineral density and inflammatory bowel disease severity. Brazilian Journal of Medical and Biological Research 201750 e6374. (https://doi.org/10.1590/1414-431X20176374)

25 Cravo M, Guerreiro CS, dos Santos PM, Brito M, Ferreira P, Fidalgo C, Tavares L \& Pereira AD. Risk factors for metabolic bone disease in Crohn's disease patients. Inflammatory Bowel Diseases 201016 2117-2124. (https://doi.org/10.1002/ibd.21297)

26 Abraham BP, Prasad P \& Malaty HM. Vitamin D deficiency and corticosteroid use are risk factors for low bone mineral density in inflammatory bowel disease patients. Digestive Diseases and Sciences 201459 1878-1884. (https://doi.org/10.1007/s10620-014-3102-x)

27 Héla S, Nihel M, Faten L, Monia F, Jalel B, Azza F \& Slaheddine S. Osteoporosis and Crohn's disease. Joint Bone Spine 200572 403-407. (https://doi.org/10.1016/j.jbspin.2004.09.006)

28 Bryant RV, Ooi S, Schultz CG, Goess C, Grafton R, Hughes J, Lim A, Bartholomeusz FD \& Andrews JM. Low muscle mass and sarcopenia: common and predictive of osteopenia in inflammatory bowel disease. Alimentary Pharmacology and Therapeutics 201541 895-906. (https:// doi.org/10.1111/apt.13156)

Received in final form 24 June 2021

Accepted 14 July 2021

Accepted Manuscript published online 14 July 2021
This work is licensed under a Creative Commons Attribution-NonCommercial 4.0 International License. ded from Bioscientifica.com at 04/26/2023 01:02:11PM 